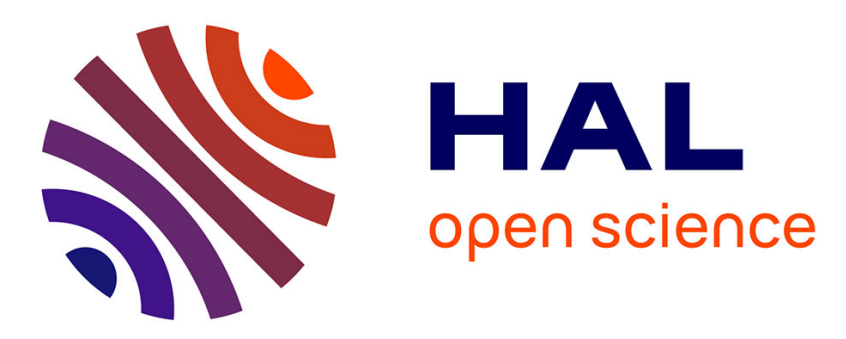

\title{
La co-production de la performance arbitrale de haut niveau : le cas du rugby
}

Géraldine Rix-Lièvre, Simon Boyer, Fatia Terfous, Fabien Coutarel, Pascal Lièvre

\section{To cite this version:}

Géraldine Rix-Lièvre, Simon Boyer, Fatia Terfous, Fabien Coutarel, Pascal Lièvre. La co-production de la performance arbitrale de haut niveau: le cas du rugby. Sociologie du Travail, 2015, 57, pp.496515. 10.1016/j.soctra.2015.09.005 . hal-01225694

\section{HAL Id: hal-01225694 \\ https://hal.science/hal-01225694}

Submitted on 10 Jan 2018

HAL is a multi-disciplinary open access archive for the deposit and dissemination of scientific research documents, whether they are published or not. The documents may come from teaching and research institutions in France or abroad, or from public or private research centers.
L'archive ouverte pluridisciplinaire HAL, est destinée au dépôt et à la diffusion de documents scientifiques de niveau recherche, publiés ou non, émanant des établissements d'enseignement et de recherche français ou étrangers, des laboratoires publics ou privés. 


\title{
La co-production de la performance arbitrale de haut niveau : le cas de l'arbitrage en rugby
}

\author{
Géraldine Rix-Lièvre \\ Laboratoire ACTé (EA 4281), Clermont Université, Université Blaise Pascal, UFR STAPS, \\ BP 10448, F-63000 Clermont-Ferrand \\ Geraldine.rix@univ-bpclermont.fr
}

\section{Simon Boyer}

Laboratoire ACTé (EA 4281), Clermont Université, Université Blaise Pascal \& Groupe La Poste, BP 10448, F-63000 Clermont-Ferrand

Simon.boyer@univ-bpclermont.fr

\section{Fatia Terfous}

Laboratoire ACTé (EA 4281), Clermont Université, Université Blaise Pascal, BP 10448, F63000 Clermont-Ferrand

Fatia.Terfous@univ-bpclermont.fr

\section{Fabien Coutarel}

Laboratoire ACTé (EA 4281), Clermont Université, Université Blaise Pascal, UFR STAPS, BP 10448, F-63000 Clermont-Ferrand

Fabien.coutarel@univ-bpclermont.fr

\section{Pascal Lièvre}

Laboratoire CRCGM (EA 3849), Clermont Université, Université d'Auvergne, 
BP 10448, F-63000 Clermont-Ferrand

PascalLievre@orange.fr

\section{Correspondance :}

Géraldine Rix-Lièvre,

geraldine.rix@univ-bpclermont.fr

Tel : 0473407855 // Fax : 0473407446

\section{Déclaration d'intérêts}

Les recherches dont cet article rend compte ont été financées par le Conseil Régional d'Auvergne et le PERF Arbitrage (http://perf.arbitrage.univ-bpclermont.fr/). Les auteurs déclarent ne pas avoir de conflit d'intérêts en relation avec cet article.

\section{Remerciements}

Les auteurs remercient la Fédération Française de Rugby d'avoir autorisé et accompagné les recherches présentées dans cet article ; les arbitres, les superviseurs et les joueurs d'y avoir contribué. Ils remercient également les coordinateurs de ce numéro spécial et les évaluateurs anonymes de la revue qui, par leurs commentaires, ont permis d'enrichir et d'améliorer ce texte. 
Résumé : Si l'arbitrage établit la performance sportive, l'arbitre de haut niveau doit lui aussi produire une performance. Cet article interroge les conditions de sa construction en étudiant le cas de l'arbitrage en rugby. Nous établissons que la performance arbitrale procède, sur le terrain, d'une co-production. Nous montrons tout d'abord en quoi les modes de coordination entre l'arbitre et les joueurs participent de la performance produite. Ensuite, nous interrogeons la manière dont cette prestation est évaluée. Deux modalités d'évaluation pèsent sur l'arbitrage de haut-niveau: une évaluation officielle institutionnelle produite par un superviseur, une évaluation publique proposée par la presse. Leur investigation met en évidence la variabilité et la disparité des critères d'évaluation de la performance arbitrale de haut-niveau.

Mots-clés : arbitre, activité, évaluation, performance, rugby, sport. 


\section{La co-production de la performance arbitrale de haut niveau : \\ le cas de l'arbitrage en Rugby.}

Le système sportif s'est construit en standardisant le cadre spatio-temporel de pratique, en adoptant des règles du jeu formalisées et en plaçant l'organisation de la pratique sous l'égide d'une institution (Arnaud, 1995; Darbon, 2008). Ce mouvement s'est accompagné d'une rationalisation et d'une quantification des techniques du corps : la performance sportive devait être reproductible et établie de manière objective. Afin d'établir cette performance et d'assurer une réalisation conforme au cadre posé par les règles, des juges ou des arbitres ont été introduits. L'arbitrage peut ainsi être considéré comme un élément constitutif de la performance sportive en ce qu'il l'établit. Mais l'arbitre de haut niveau doit lui aussi produire une performance. A l'instar de n'importe quel sportif, il s'inscrit dans un système compétitif où ses prestations sur le terrain sont scrutées et évaluées; des appréciations et des évaluations dont dépend son avenir dans le sport de haut niveau. Si les conditions et les modalités d'établissement de la performance sportive paraissent réglées, objectivées et socialement établies, tel n'est pas le cas de la performance arbitrale. Il est même rare que tout le monde s'accorde sur la qualité de l'arbitrage. Dans le cadre de cet article, nous interrogeons les modalités de production et de construction d'une performance arbitrale de haut niveau en partant du cas de l'arbitrage en rugby. Ce cas s'est imposé pour plusieurs raisons. La première tient au fait que le rugby est un sport populaire, professionnel et médiatisé où la performance arbitrale est scrutée et s'inscrit dans un contexte où les enjeux symboliques, économiques et sociaux sont importants. La seconde renvoie à la manière dont l'arbitrage en rugby est spontanément perçu. En première approximation l'arbitre semble, plus qu'ailleurs, influencer le déroulement du jeu : (1) lors de certaines phases de jeu, selon la règle qu'il mobilise, il peut siffler dans un même contexte pour l'une ou au contraire l'autre équipe ; (2) la diffusion 
télévisuelle des propos de l'arbitre en match montre qu'il intervient au cours du jeu. La troisième, plus méthodologique, est probablement la plus importante. Investir les pratiques de haut-niveau suppose une pleine intégration du chercheur dans le système : c'est un travail de terrain entrepris il y a plus de dix ans qui a permis de construire les bases d'une véritable collaboration et a ouvert la possibilité d'étudier les pratiques d'arbitrage de haut-niveau en Rugby.

Dans une première partie, nous montrons que les études s'intéressant à la performance arbitrale apportent une définition fragmentée -même si elle se veut exhaustive- de la performance de l'arbitre. Nous proposons d'opérer une rupture avec ces dernières afin de prendre en compte la complexité de l'activité de l'arbitre telle qu'elle se déroule effectivement en match. Cette orientation amène à considérer que la performance arbitrale ne peut être réduite à la prestation d'un individu, l'arbitre, mais que cette dernière est largement co-produite dans une coordination avec les joueurs. Par conséquent, nous proposons dans un second temps d'étudier les modalités de co-production de la prestation arbitrale sur le terrain au cours d'un match. Cette étude renseigne ainsi la performance arbitrale telle qu'elle est coproduite. Mais une prestation n'est performance que si elle est désignée comme telle par un jugement extérieur. En effet, un arbitre n'est - et reste- arbitre de haut niveau que si le système sportif reconnaît sa compétence à arbitrer. Nous avons donc investigué deux modalités d'évaluation qui pèsent sur l'arbitrage de haut niveau : (1) une évaluation officielle institutionnelle produite par un superviseur chargé, par la fédération, d'apprécier la performance arbitrale produite sur le terrain au cours d'une rencontre, (2) une évaluation profane publique proposée par la presse. L'analyse de l'activité du superviseur d'une part et l'analyse d'un corpus de presse écrite d'autre part mettent en évidence la variabilité et la disparité des critères d'évaluation et des attentes qui pèsent sur la performance arbitrale de haut niveau. 


\section{Des déterminants de la prestation de l'arbitre à la co-production de la performance}

\subsection{Facteurs de performance et caractéristiques du « bon » arbitre}

La plupart des études de la performance sportive procèdent d'une analyse qui isole un certain nombre de facteurs contribuant à sa réalisation; les sciences du sport opèrent souvent un morcellement de la performance (Hauw et Durand, 2004). Celle de l'arbitre ne fait pas exception. Même si peu de travaux affichent une centration sur la performance arbitrale, la plupart portent un regard normatif sur la prestation de l'arbitre et établissent les caractéristiques et compétences $\mathrm{du}$ «bon» arbitre. Ainsi, alors même que les normes de performance pour les arbitres ne sont pas socialement objectivées et établies de manière univoque, les productions scientifiques tendent à les formaliser, participant ainsi à la construction de la performance.

Certaines études s'attachent à définir et qualifier la sollicitation physique relative à l'arbitrage selon les spécificités sportives (Coutts et Reaburn, 2000; Helsen et Bultynck, 2004; Kay et Gill, 2003, 2004; Krustrup et al., 2009). Dans ce domaine, les recherches se multiplient d'autant plus que les fédérations se dotent progressivement de «tests physiques » pour évaluer et différencier les arbitres. La non-atteinte des minima signifie pour les arbitres concernés un changement de niveau pour la saison en cours. Les études sur l'activité physique en match contribuant à une meilleure connaissance des sollicitations physiques fondent la pertinence et la légitimité des tests. Ainsi, la performance arbitrale est rapportée à une performance physique souvent avec l'idée sous-jacente qu'un arbitre, pour bien juger, doit être bien placé et donc avoir certaines aptitudes physiques pour se déplacer et se placer correctement. 
Cette idée est partagée par une partie des travaux sur la prise de décision, notamment ceux mettant en évidence les biais perceptifs qui peuvent tronquer le jugement de l'arbitre (Catteeuw et al., 2010; Oudejans et al., 2005). Ils établissent notamment que l'arbitre doit être bien placé pour bien voir la situation et juger correctement de la faute relativement aux règles du jeu. Ainsi, un second critère de performance apparaît: l'exactitude des décisions par rapport à une « bonne application du règlement» (Mascarenhas et al., 2005; Plessner et Haar, 2006). La performance arbitrale repose ainsi sur la capacité de l'arbitre à prendre les bonnes décisions. De plus, une bonne communication (Mellick et al., 2005) est aussi un facteur de performance, comme la gestion du stress et le sentiment d'efficacité (Guillén et Feltz, 2011). Au-delà des capacités physiques, des compétences sont donc requises en termes de traitement de l'information, de prise de décision, de gestion du stress, de communication (Ollis et al., 2006).

Ces études identifient des facteurs de performance de natures différentes : ce sont autant d'aptitudes, de capacités, de compétences dont l'arbitre doit faire preuve et qui semblent conditionner sa prestation. Ces études qui aboutissent à un morcellement de la performance ont en commun une conception de l'arbitrage qui s'inscrit dans un formalisme juridique (RixLièvre et al., 2014). Celui-ci peut être décliné en trois points : (1) la faute est factuelle, (2) la règle détermine les jugements, (3) la tâche de l'arbitre est de bien connaître la règle et de bien voir les faits. Mais d'autres recherches montrent qu'il est impossible de réduire la performance arbitrale à l'identification des fautes ; elle est aussi relative au management du jeu, à l'équité et à la cohérence des prises de décision ou au maintien de la sécurité (Mascarenhas et al., 2006). En effet, dès que l'activité de l'arbitre est replacée dans son contexte, la question de sa performance apparait plus complexe : « refereeing at the top level is about "finding a set of solutions that work for you on the day" » (Ibid., 2006, p112). Il semble difficile de réduire une performance arbitrale de haut niveau à la production mécanique d'une application de la règle 
assurée par des capacités physiques d'une part, cognitives et émotionnelles d'autre part. « La performance est un tout complexe » qu'il s'agit d'appréhender en contexte ; pour ce faire, il est nécessaire de s'intéresser à l'activité, c'est-à-dire à « ce que fait un athlète en compétition, et non à ce qui est supposé déterminer ce qu'il fait » (Hauw, Durand, 2004, p122).

\subsection{Une approche de l'activité de l'arbitre en match}

Quelques travaux ont étudié l'arbitrage dans son contexte quotidien de pratique (MacMahon, 1999; Mascarenhas et al., 2005; Ollis et al., 2006). Certains se centrent sur les caractéristiques du Naturalistic Decision Making des arbitres (MacMahon, 1999; Mascarenhas et al., 2005), d'autres sur les modalités de construction de l'expertise (Ollis et al., 2006), mais peu se sont effectivement intéressés à l'activité de l'arbitre comme objet scientifique. Dans le sillage des théories de l'activité, nous proposons d'emprunter la voie initiée par le «practice turn » (Licoppe, 2008). Ancrée dans un travail ethnographique, cette perspective s'attache à «appréhender la performance dans sa globalité et à recueillir des données in situ » (Sève et al., 2006, p48) pour comprendre la manière dont, dans un contexte particulier, un arbitre construit sa situation, afin d'approcher ses modes de raisonnement pratique ordinaire ${ }^{1}$. Cette approche de l'activité de l'arbitre en match a permis de caractériser ce que fait l'arbitre au moment même où il arbitre et d'étudier cette pratique relativement à ce qu'elle produit. Ses coups de sifflet, propos et gestes ne sont plus considérés comme le résultat prévisible d'une analyse de la conformité du jeu par rapport au règlement, mais comme des actes de jugement qui montrent et imposent aux joueurs ce qui est possible à un moment donné. Ces premiers travaux sur l'activité de l'arbitre établissent que chaque acte de

\footnotetext{
${ }^{1}$ Les modalités selon lesquelles les théories de l'activité ont permis de renouveler les perspectives scientifiques sur l'arbitrage ont fait l'objet d'autres publications (voir Rix, 2005 ; Rix-Lièvre et al., 2014).
} 
jugement décrit de façon particulière l'activité des joueurs comme un fait, une dynamique ou un problème ; cette description est performative au sens où elle fait advenir ce qu'elle décrit, de la manière dont elle le décrit. Autrement dit, la faute n'est plus relative à la réalité d'un fait, mais dépend du rapport de l'arbitre aux actions des joueurs. Dans un même contexte de jeu, selon que l'arbitre appréhende l'activité des joueurs comme un fait, une dynamique ou un événement qui pose question, la faute ne sera pas la même. Comme le souligne Serres (2010, pp. 14-15), «c'est donc une erreur [...] que de dire : le but ou l'essai y était ou n'y était pas et l'arbitre l'a accepté ou refusé. Non le but y est dès lors que l'arbitre l'a ainsi décidé. [...] Car un but est un événement juridique décidé, mieux, créé même par l'arbitre ». Il ne s'agit pas de considérer que l'arbitre fait le jeu ou invente des buts, mais de souligner que même si l'activité des joueurs préexiste aux actes de jugement de l'arbitre, c'est la manière dont l'arbitre la montre qui la fait advenir en tant qu'événement du jeu -but, essai, faute... Ainsi, l'arbitre co-produit, avec les joueurs, le déroulement du jeu et les règles ne déterminent plus son activité, mais permettent, au sens de pouvoir et de possibilité, cette co-production.

Cette étude de l'activité des arbitres en match, en partant de leur expérience en situation, met en exergue l'écart important entre ce qui est souvent considéré comme la tâche de l'arbitre -appliquer des règles- et son activité effective. Ainsi, la qualité de la prestation de l'arbitre ne peut être rapportée au simple critère formel d'application de la règle. Si l'arbitrage consiste à co-produire avec les joueurs le déroulement du jeu, il devient alors impossible d'isoler la performance produite par l'arbitre de la prestation des joueurs. Comme dans d'autres métiers dans le domaine des services (Motté et Haradji, 2007; Valléry et Leduc, 2005), la performance est largement co-produite. Nous proposons donc de re-questionner la performance arbitrale pour prendre en charge sa complexité et son caractère co-produit. 


\section{La performance arbitrale : une co-production sur le terrain}

\subsection{Etudier la coordination joueurs-arbitre}

Nous avons tout d'abord étudié l'activité de l'arbitre et ses modes de coordination avec les joueurs en match. Cette étude fine de l'activité des acteurs participant directement à la réalisation de la performance éclaire l'arbitrage tel qu'il se déroule effectivement sur le terrain. Suivant une perspective ancrée dans les théories de l'activité, nous avons examiné la coordination entre les joueurs et les arbitres en prenant en compte la manière dont chacun vit sa situation (Poizat et al., 2012). L'objectif était de « saisir empiriquement la manière dont les personnes s'orientent dans les situations et les ressources dont elles disposent pour le faire au fil de leur activité » (Licoppe, 2008, p296). Si ce que l'arbitre montre et impose aux joueurs dépend de la manière dont il appréhende leur activité, ses modalités de coordination avec les joueurs sont également relatives à la manière dont ils construisent leurs situations. Nous avons donc étudié de concert, l'expérience de l'arbitre en match lorsqu'il montre quelque chose aux joueurs, et les expériences de ces derniers.

Quatre matches du championnat élite (Top 14-Orange) ont été investigués au cours des saisons $2009 / 10$ et $2010 / 11^{2}$. Pour approcher ce qui est significatif pour un arbitre tout au long du match, nous l'avons invité à expliciter le décours de son activité à partir de

\footnotetext{
${ }^{2}$ Quatre arbitres ont participé à ce travail ainsi que seize joueurs issus de quatre équipes différentes. Lors de chaque rencontre, plusieurs enregistrements vidéo ont été produits (1) un en plan large depuis les tribunes couplé au son issu du « micro-arbitre », réalisé par les diffuseurs du match à la demande de l'équipe de recherche ; (2) un autre à partir d'une caméra embarquée filmant une perspective proche de celle de l'arbitre en situation. Cette perspective dite subjective située est construite en équipant l'arbitre d'une caméra (Ø 8mm) fixée au niveau de sa tempe et d'un système d'enregistrement embarqué (trois boîtiers de respectivement $8 \times 5 \times 2 \mathrm{~cm}, 9 \times 5 \times 1,5 \mathrm{~cm}$, $7,5 \times 6,5 \times 2 \mathrm{~cm}$, pesant au total $350 \mathrm{~g}$ ) placé dans la ceinture utilisée pour porter les appareils de transmission audio. Ces traces d'activité ont été mobilisées a posteriori pour mener différents entretiens individuels, -un avec l'arbitre, quatre avec des joueurs- afin d'approcher le vécu, ce qui est significatif, ce qui importe pour chacun en situation. Différentes méthodes se donnent cet objectif (Cahour et Licoppe, 2010; Theureau, 1992; Vermersch, 1994). Dans cette recherche, nous avons opté pour des entretiens utilisant des enregistrements vidéo comme support. En effet, pour saisir ce qu'un acteur a effectivement vécu dans un moment particulier, l'utilisation d'une vidéo comme trace d'activité présente deux avantages: "(1) spécifier le moment dont il est question, (2) constituer une trace dynamique du déroulement de la pratique » (Rix-Lièvre, 2010, p366).
} 
l'enregistrement de sa perspective subjective située, menant ainsi avec lui, un entretien en re situ subjectif. Il s'agit d'utiliser une perspective subjective située pour re-placer l'acteur, lors de l'entretien, dans une perspective proche de son point de vue en situation. Cette trace vise à faciliter tant l'effort de ré-flexion de l'acteur que la conduite - par le chercheur- de l'entretien vers une explicitation de l'action. Ce dispositif méthodologique utilisé pour étudier différentes pratiques est un outil de constitution de l'expérience permettant de mettre à jour des dimensions cognitives de l'activité (Rix et Biache, 2004 ; Rix-Lièvre, 2010). Pour solliciter les joueurs, le dispositif d'auto-confrontation a été retenu (Theureau, 1992). C'est donc à partir d'une sélection d'extraits de l'enregistrement du match d'un point de vue extérieur, des tribunes et en plan large, que certains joueurs ${ }^{3}$ ont été invités à expliciter leurs vécus dans les moments présentés par la vidéo. Les verbalisations issues de ces entretiens ont ensuite été retranscrites et traitées de concert avec des matériaux d'observation dans le but de formaliser certains déterminants cognitifs de la coordination joueurs-arbitre en partant de l'expérience dévoilée. Nous avons en particulier examiné ce qui est saillant et significatif d'une part pour l'arbitre, d'autre part pour un joueur lors d'un même moment de match. Nous avons construit des tableaux mettant en parallèle une description du contexte, les verbalisations de l'arbitre relatives à ce moment et celles des joueurs.

\subsection{Une coordination malgré un partage cognitif très limité}

Dans la mesure où le partage de «contenus cognitifs » est souvent considéré comme une condition favorisant la coordination (Bourbousson et al., 2011; Salembier et Zouinar,

3 Etant dans l'impossibilité matérielle de travailler avec l'ensemble des joueurs du match, nous en avons sélectionné quatre. Nous avons privilégié, pour des questions de faisabilité, la participation de l'équipe jouant à domicile. Parmi l'ensemble des joueurs ayant pris part au match, nous en avons sélectionné quatre en fonction de leurs statuts (demi-de-mêlée, capitaine, joueur avant ou arrière) et de leurs interactions patentes avec l'arbitre, que ce soit dans le jeu ou pendant les arrêts de jeu. Ce faisant, l'identification des joueurs participant à l'étude n'a été effectuée qu'une fois le match terminé. Selon leurs clubs d'appartenance, les joueurs ont été mis à la disposition du chercheur vingt à trente minutes en fonction de leur programme d'entrainement. 
2004), nous avons cherché à mettre en regard, dans des moments spécifiés, la manière dont l'arbitre d'une part, certains joueurs d'autre part construisent spontanément leurs situations. Nous avons relevé en particulier les éléments saillants et les préoccupations de chacun.

Au cours d'un match de rugby, de multiples actions se déroulent simultanément. Les éléments de contexte saillants pour l'arbitre et pour les joueurs sont le plus souvent distincts. Lors d'un ruck, ou mêlée spontanée ${ }^{4}$, l'arbitre pointe les joueurs non-participants à la lutte pour le ballon, il leur indique : « Reculez, reculez ! » pour ne pas qu'ils soient hors-jeu ${ }^{5}$. Pour l'arbitre, «Là [dans le regroupement] le ballon est propre, il faut les retenir [ceux qui sont au large] "Reculez, reculez" pour ne pas qu'ils fassent la faute ». Mais pour le joueur qui participe au regroupement, c'est l'adversaire qui entre dans le ruck qui est saillant : « Là, là on était bien et le 5 Noir $^{6}$, vient là, là retarder [la sortie] ». L'arbitre et le joueur sont focalisés sur des éléments de contexte différents. Et lorsque ce qui est saillant pour les uns et les autres se rapporte aux mêmes éléments de contexte, les joueurs ne l'appréhendent souvent pas de la même manière que l'arbitre. Par exemple, lors d'une mêlée dans le camp des rouges introduction rouge, l'arbitre marque l'emplacement de la mêlée ${ }^{7}$ et dit « Là elle est là ». Les deux packs $^{8}$ se mettent en place. L'arbitre dit « Je m'enlève », recule, se baisse en continuant « Flexion, Touchez, Stop, Entrez ». La mêlée se forme, le pack rouge avance, puis est chahuté et recule sur le côté, la mêlée s'effondre, l'arbitre siffle pénalité au bénéfice des noirs, puis indique «C'est la poussée...». Le 8 rouge s'approche de l'arbitre qui lui précise «Vous ramassez la balle dans la mêlée ». Ce qui est saillant pour l'arbitre c'est qu'il y a : « un

\footnotetext{
${ }^{4}$ Le ruck ou mêlée spontanée est une situation d'affrontement physique au sol faisant suite à une phase de plaquage, opposant un plaqueur sans ballon et un plaqué portant le ballon. Au cours de cette phase de jeu, alors que le ballon est au sol, un ou plusieurs joueurs de chaque équipe sur leurs pieds luttent au corps à corps pour le gain/la conservation du ballon en faisant reculer l'adversaire et/ou en venant talonner le ballon.

${ }^{5}$ La ligne de hors jeu lors d'une phase de mêlée spontanée -ou ruck- est une ligne imaginaire parallèle à la ligne d'en-but, passant par les pieds des derniers participants à la phase de jeu au sol. C'est une ligne au-delà de laquelle les défenseurs n'ont pas le droit, tant que le ballon est dans le regroupement, de faire action de jeu.

${ }^{6}$ Afin d'assurer, malgré la renommée de certains, l'anonymat des joueurs et des arbitres, toutes les couleurs de maillots ont été uniformisées et par convention, nous opposerons les joueurs noirs aux rouges.

${ }^{7}$ Lors de chaque mêlée, l'arbitre trace au pied un trait déterminant l'emplacement de cette dernière. Selon la règle, chaque équipe doit se placer de part et d'autre de ce trait à environ 50 centimètres.

${ }^{8}$ Le pack constitue les 8 joueurs de chaque équipe qui participent à la mêlée.
} 
premier impact des rouges mais après les noirs se ressaisissent et prennent l'ascendant. Et c'est au moment où ils prennent l'ascendant que je me retrouve avec le huit qui va être couché complètement en-dessous. Le huit rouge... Voilà tu vois ils avancent, et là t'as un tas avec le huit rouge dessous. Pour moi la poussée, elle est noire et pour assurer le gain du ballon, le huit a dû aller chercher la balle dans la mêlée, ce qu'il n'a pas droit de faire, avec la main. Je mets la pénalité pour les noirs ». Ce qui est saillant pour le 8 rouge, c'est aussi ce double mouvement et la mêlée qui s'effondre, mais la signification qu'il confère à ce double mouvement et à cet effondrement est différente : «on sent qu'il y a une avancée [...]. Au début ça avance, donc très bien, le ballon il commence à sortir. Et au moment où je vais pour ramasser le ballon les mecs ils rebalancent, du coup on prend la... notre pilier il se fait, il se fait un peu relever, du coup tout tombe sur moi...». Que les éléments de contexte saillants pour l'arbitre et pour le joueur soient ou non en partie partagés, ce qui est significatif pour chacun se distingue largement de ce qui l'est pour l'autre -pour l'arbitre, le 8 rouge fait écrouler la mêlée en passant dessous pour prendre le ballon à la main alors qu'il recule ; pour le 8 rouge, la poussée ascendante du pilier adverse disloque la mêlée qui lui tombe dessus ; ils vivent des situations très différentes.

De même les préoccupations des joueurs sont souvent largement à l'écart de celles de l'arbitre. Par exemple, lors de la seconde mêlée du match, l'arbitre marque l'emplacement de la mêlée en traçant avec le pied une ligne au sol, les joueurs se rapprochent. Les deux

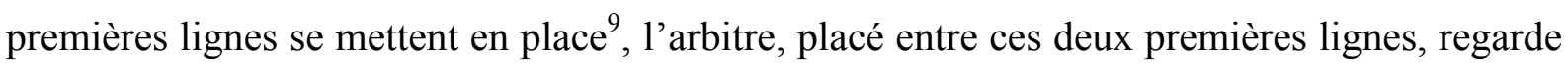
à droite, à gauche et dit : «On attend l'annonce ». Il recule. A coté de la mêlée, il annonce : «Flexion, touchez, stop, entrez... Voilà ». La mêlée se forme, le 9 noir introduit le ballon, les rouges avancent. Ce qui préoccupe l'arbitre à ce moment là c'est de bien mettre en place la mêlée pour qu'il n'ait pas à siffler : " "On attend l'annonce", je remets une couche sur les

\footnotetext{
${ }^{9}$ Les trois joueurs de premières lignes se placent près de la ligne tracée, face aux adversaires et se lient entre eux.
} 
rouges pour leur remettre la pression... [...] Là, j'attends de voir, je regarde qu'ils soient 5 [...] Tant que j'ai pas les secondes lignes... alors les noirs, là, ils viennent plus tard que les rouges. [...] Les secondes lignes vont s'y mettre, comme les rouges, ils s'y sont mis. Je me fixe sur les noirs et là j'annonce mon commandement. "Voilà", c'est juste pour les rassurer, et peut-être me rassurer moi...». De son côté, le joueur de première ligne rouge s'attache à oublier la première mêlée -lors de laquelle son équipe a été pénalisée- pour reprendre l'ascendant sur le pack adverse : «c'est deuxième mêlée, la première mêlée on l'a oubliée, on essaie de passer à autre chose... [...] Là, c'est bien rentré, on est resté compact, on avance un peu là sur... leur introduction. Donc voilà, c'est ça c'est important, on les enfonce, donc ça, ça met en confiance pour la suite ». L'exemple précédent expose un cas où les préoccupations de l'arbitre et du joueur considéré sont différentes, ce que nous avons constaté dans la majorité des cas. Ce résultat n'est pas surprenant au regard des objectifs et des rôles spécifiques de chacun au cours d'un match. Mais nous constatons également que l'absence de compréhension partagée ne se traduit dans les cas présentés, ni par une impossibilité de coordination, ni par de longues discussions : elle n'affecte pas dans l'instant le déroulement du match.

\subsection{Ce que montre l'arbitre : entre contraintes et ressources de la co-production du déroulement du jeu}

Afin d'approcher les différentes modalités de coordination entre les joueurs et l'arbitre, nous nous sommes alors centrés sur l'expérience des joueurs pour envisager la manière dont ce que montre l'arbitre s'impose effectivement, c'est-à-dire ce qui les conduit à agir fidèlement à ce que l'arbitre montre et impose. Nous nous sommes donc intéressés à la manière dont en match, les joueurs appréhendent ce que montre l'arbitre. Nous avons 
distingué trois cas qui révèlent différentes modalités de coordination : ce que montre l'arbitre s'impose sur le mode de la résignation, de l'évidence ou repose sur une réflexivité-en-acte du joueur.

A différentes reprises, ce que vit le joueur est en contradiction avec ce que montre l'arbitre, mais il s'exécute dans la mesure où « on peut rien y faire alors... », « il est seul juge, c'est lui qui décide ». Par exemple, lors d'un engagement rouge, les noirs récupèrent le ballon, après une phase de jeu au sol un noir sort le ballon et joue au pied dans le camp adverse, un rouge récupère le ballon, il est plaqué, trois joueurs arrivent dans la zone plaqueur/plaqué et viennent lutter pour le gain du ballon. L'arbitre court en direction de cette zone. Il siffle pénalité, montre un joueur noir et indique : «entrée latérale ». Les joueurs noirs lèvent les bras au ciel et repartent se replacer à 10 mètres. Pour l'arbitre : « C'est le noir sur le côté », pour lui c'est un fait incontestable, il rentre sur le côté ${ }^{10}$. La perception d'un des joueurs noirs ayant participé à la phase de jeu est différente : « elle est à nous, parce que on avance sur le contact, on les fait reculer, on a deux joueurs qui grattent le ballon. Il nous siffle un peu c'te faute... pour moi, y a pas faute. Mais après c'est, après c'est l'arbitre qui choisit et puis voilà ! ». Le joueur considère la dynamique de l'activité des joueurs qui arrivent dans le regroupement, alors que l'arbitre ne voit qu'un fait: le joueur noir entre sur le côté, il sanctionne. Dans ce cas, il y a une absence totale de compréhension partagée du contexte de jeu. Comme le soulignent d'autres études, le joueur semble alors se plier à « une décision émanant d'un tiers avec lequel il est dans une relation hiérarchique dissymétrique défavorable et face à laquelle il n'a aucun recours » (Reynes et al., 2008, p11). Ainsi, la position de pouvoir de l'arbitre par rapport au joueur semble assimilée et il ne cherche pas à contester malgré «l'injustice perçue, et des sentiments de dépit/résignation» (Ibid., p11). Dans

\footnotetext{
${ }^{10}$ Lors d'un ruck ou mêlée spontanée, les joueurs peuvent venir à la lutte pour le gain du ballon s'ils arrivent dans l'axe du regroupement c'est-à-dire selon un axe perpendiculaire à la ligne de but, qui passe par le regroupement. S'ils arrivent par le côté du regroupement, ils sont pénalisables.
} 
l'exemple présenté, le joueur noir s'exprime dans ce sens : «C'est frustrant, moi j’ai tendance à faire voir un peu tout ça.... Mais j'essaie même pas de lui [l'arbitre] faire voir parce que... ça, c'est en plus le genre de geste qui peut mettre à dos l'arbitre ou le contrarier ». Dans ce cas, ce que montre l'arbitre s'impose au joueur sur le mode de la résignation qui s'accompagne d'un sentiment de frustration.

L'étude de Reynes et al. (2008) tend à montrer que ces sentiments de frustration et de résignation sont à l'origine de réactions agressives des joueurs envers l'arbitre ou d'autres joueurs. Cependant, il n'y a pas toujours, de la part des joueurs, une absence totale de compréhension ou d'acceptation d'un point de vue différent puisque ce que montre l'arbitre peut aussi s'imposer selon d'autres modalités. Nous avons distingué des moments au cours desquels ce que montre l'arbitre s'impose réflexivement. Même si les éléments de contexte saillants pour le joueur sont à l'écart de ce que montre l'arbitre, il considère que ce dernier a dû prendre en compte d'autres éléments. Par exemple, dans le contexte suivant: les rouges ont la possession du ballon et progressent en direction de l'en-but adverse, le 9 et le 7 noirs plaquent le porteur du ballon, le 1 noir accompagne le mouvement au sol au niveau des épaules du joueur plaqué. L'arbitre suit le mouvement juste derrière eux. Puis deux rouges viennent à la lutte pour déblayer les noirs ${ }^{11}$. L'arbitre siffle et pointe les joueurs au sol puis il annonce : « 7 noir !». Pour l'arbitre : « Le 7 est resté... il fait aucun effort de se sortir, il reste sur le ballon... y a pas de continuité possible donc, pénalité. [...] C'est-à-dire pour moi, il a pas fait l'effort, il a pas bougé, c'est lui qui empêche la sortie... ${ }^{12}$. Pour le 1 noir, ce qui est prégnant est totalement différent : «En fait moi je suis sur mes appuis, j’ai le droit de gratter le ballon, mais il dit... au début, c'est de l'étonnement parce que je crois qu'il me pénalise moi. Et après, il annonce que c'est l'autre joueur qui est pénalisé parce qu'il plonge. Voilà,

\footnotetext{
${ }^{11}$ C'est-à-dire les repousser hors de la zone plaqueur/plaqué.

${ }_{12}$ Au rugby, la règle impose au plaqué de lâcher le plaqueur et de faire l'effort de sortir de la zone plaqueur/plaqué.
} 
c'est là qu'il annonce. C'est un peu d'étonnement parce que moi je suis le premier défenseur à gratter le ballon, et ça lâche pas à ce moment là. [...] Et quand je me relève, je vois qu'il me montre du doigt, donc je croyais que c'était moi [...] Quand il annonce le 7, donc après j'ai pas trop compris, il dit ben "il plonge sur le côté", j’avais compris que... qu'il était pas resté sur ses appuis ». D'une part, cet extrait d'entretien met en évidence l'absence d'une compréhension partagée de la situation au moment où l'arbitre siffle : ce qui est significatif pour le 1 noir - «j'ai le droit, je suis sur mes appuis »- est à l'écart de ce que montre l'arbitre ; l'incompréhension est même patente. D'autre part, les propos du joueur permettent d'identifier le processus à l'œuvre en situation une fois que l'arbitre a sifflé : malgré la prégnance de ce qu'il a vécu, ce que montre l'arbitre l'amène à reconstruire un point de vue différent qui est compréhensible ${ }^{13}$. Il n'apparait pas évident mais il est acceptable au sens où le joueur en construit réflexivement la logique. Ainsi, la réflexivité-en-acte des joueurs fonde une autre modalité de coordination : le joueur se conforme à ce que l'arbitre montre et impose dans la mesure où il accepte la description de l'arbitre comme une « autre manière de voir les choses » qu'il rapporte à la perspective d'observation singulière de l'arbitre, à la convocation d'une autre règle... In fine, même si la logique qu'il reconstruit -le 7 plonge et n'est pas sur ses appuis-n'est pas celle qui préside à ce que l'arbitre montre -le 7 ne sort pas de la zone de plaquage-, la réflexivité-en-acte du joueur lui permet, alors qu'il se replace sur le terrain, de considérer que ce que montre l'arbitre est justifiable.

Cette réflexivité-en-acte, en lieu et place de la frustration, pourrait être le creuset potentiel non de comportements agressifs, mais d'une véritable compréhension de ce qui, pour l'arbitre, est acceptable dans le jeu. Si cette réflexivité-en-acte relève de l'activité du joueur, l'arbitre la suscite plus ou moins selon la manière dont il rend lisible ce qui est

\footnotetext{
${ }^{13}$ Cette reconstruction n'est rendue possible que dans la mesure où le jeu est arrêté. L'ensemble des actions et interactions que nous relatons se déroulent en quelques secondes et l'opposition entre les joueurs au cours de cette phase de jeu au sol est très intense. Ce n'est qu'une fois le jeu arrêté que le joueur construit réflexivement la logique de l'activité de l'arbitre.
} 
acceptable/inacceptable pour lui. Les descriptions qu'il fait des situations, les explications/justifications éventuelles qu'il produit, offrent autant d'opportunités aux joueurs de construire la logique et la légitimité de ce qui est montré. Ainsi, le fait que l'arbitre prenne, dans certains cas, un temps pour rendre compte de son processus de décision semble être un levier de la performance arbitrale (Mellick et al., 2007; Rix-Lièvre et al., 2014). Les réponses apportées par l'arbitre aux questions des joueurs ou des excuses faites en cas d'erreur favorisent le respect de l'arbitre, respect qui améliore la perception d'équité ; cette équité perçue et ce sentiment de justice contribuent aux comportements positifs des joueurs (Simmons, 2007). Mais en-deçà des explications/justifications que l'arbitre produit, ses interventions constituent aussi une ressource pour les joueurs dans la compréhension du sens, de la logique, de ce qu'il montre et impose. Par exemple, suite à un engagement des noirs, plusieurs joueurs des deux équipes luttent dans les airs pour le gain du ballon, celui-ci-est projeté en direction de la ligne d'en-but noire, l'arbitre dit «Derrière, jouez » ${ }^{14}$. Pour l'arbitre : «"Y a rien, jouez" j'ai dit : "elle est derrière, jouez" ». En indiquant « Derrière, jouez », il décrit la manière dont il vit la situation et établit que le jeu qui se déroule est acceptable. Pour un des joueurs rouges : «Ouais c'est entendu ça. Ça c'est bien parce que quand y a des doutes comme ça au moins ça laisse pas... [...] donc il dit "y a rien, jouez". Donc là de suite les joueurs... [...] donc c'est important qu'il nous dise comment lui il a apprécié le truc quoi, comment il le voit ». En stipulant « Jouez, jouez », « c'est bon » ou «y a rien », l'arbitre contribue à rendre explicite non plus seulement une faute -qui est signalée par un geste, une injonction et/ou un coup de sifflet-, mais aussi ce qui est acceptable. Le jeu pourrait se poursuivre si l'arbitre n'indiquait rien, mais le fait d'imposer « Jouez, jouez » lève l'incertitude des événements et rend manifeste le fait que l'arbitre a jugé. Ainsi, ce que l'arbitre montre -la manière dont il décrit le jeu-constitue une véritable ressource pour la

\footnotetext{
${ }^{14}$ Dans ce type de contexte, une confusion peut exister sur le fait qu'il y ait ou non en-avant en fonction du (des) joueur(s) qui touche(nt) le ballon.
} 
coordination entre l'arbitre et les joueurs ; ce que confirme de manière plus générale un autre joueur : «"Ruck", là tu sais que t'as pas le droit de mettre les mains, tu peux rentrer que par l'axe et pousser, tu peux... [... Chercheur : "Ruck", c'est "c'est jugé" ?] Voilà, l'arbitre il a décidé que y avait "ruck", y a "ruck", on s'adapte au ruck. Si le mec il met la main tant pis pour lui, il savait qu'il y avait ruck. ». Ainsi, la description que fait l'arbitre du déroulement du jeu, la manière dont il qualifie tel ou tel événement constitue pour les joueurs des opportunités pour saisir le sens de ce que l'arbitre impose et s'y adapter même s'ils ne partagent pas spontanément ce qui est montré.

Si le processus de coordination précédent se fonde sur la construction explicite et réflexive d'un partage cognitif fondé sur ce que montre l'arbitre, ce partage est dans d'autres cas plus spontané. En effet, ce que montre l'arbitre peut s'imposer sur le mode de l'évidence : même si les joueurs n'appréhendent pas leur activité de la même manière que l'arbitre, ce qu'il montre leur apparaît dans l'instant comme une évidence : «j'ai rien à dire là-dessus, c'est une faute ». C'est en particulier le cas lorsque les joueurs sont pris par le jeu, centrés sur la réalisation d'une combinaison tactique ou encore perturbés par une erreur commise quelques temps de jeu avant. Par exemple, lors d'une touche dans le camp rouge, au-delà des $22 \mathrm{~m}$, le lancer revenant à l'équipe noire, le dernier sauteur noir capte le ballon, le passe à un noir, qui le transmet à son tour à un coéquipier qui est plaqué par le 7 Rouge, les noirs viennent au soutien, un noir prend le ballon dans le regroupement, et avance, il est plaqué par deux rouges. Un noir reprend le ballon dans ce nouveau regroupement et s'élance vers la ligne de but, le 7 Rouge se relève et plaque le porteur du ballon, l'arbitre qui suit l'action indique : «Hors-jeu, 7 ! » et tend le bras sur le côté signalant un avantage. Puis, il siffle. Pour l'arbitre : « Vu le 7, il a fait ça [plaquer sans se revenir dans la ligne de défense]... il a fait la faute ». En entretien, le 7 Rouge met à jour son vécu lors de la touche et indique : « En fait normalement 
je devais verrouiller le fond là. Et $[\ldots]$ je saute pas. Donc après j'étais un peu énervé ${ }^{15}$. Le jeu se poursuit, les noirs ont la possession du ballon : « donc là je voulais plaquer, directement tout ce qui bouge... Là je suis premier au plaquage, là je plaque le mec. Et après, là j'oublie de me replacer ». L'arbitre siffle, le joueur rouge reprend : «j'ai bugué je pense. [...] je veux pas qu'il passe [le joueur] ». Dans ce cas, le coup de sifflet de l'arbitre, en faisant exister un événement, rend du même coup visible son caractère inacceptable qui apparaît alors évident pour le joueur. L'entretien révèle que le joueur tout en participant au plaquage en cours reste préoccupé par sa non-réussite en touche; ce que montre l'arbitre le ramène pleinement à l'action en cours. L'intervention de l'arbitre rend manifeste certains éléments contextuels qui deviennent instantanément saillants pour le joueur et fondent un partage de significations relativement à ce que l'arbitre décrit comme acceptable/inacceptable.

Ces séquences d'activité mettent en évidence différents déterminants de la coordination joueurs-arbitre en match; les modalités de co-production du déroulement du jeu qui en découlent suggèrent que la performance arbitrale produite tient tant à l'activité de l'arbitre -la manière dont il appréhende l'activité des joueurs et dont il rend visible et lisible ce qui est acceptable/inacceptable pour lui- qu'à l'activité des joueurs -en particulier leurs rapports cognitifs à ce que l'arbitre montre. Selon leurs activités respectives, ce que montre l'arbitre ne s'impose pas de la même manière. Ces résultats éclairent ce qui se joue dans l'instant; d'autres travaux sont nécessaires pour replacer ces différentes modalités de coordination dans la temporalité du match et envisager les déterminants de la production d'une performance arbitrale dans une historicité. Cependant, notre observation participante nous a permis de relever que certains joueurs, notamment les demis-de-mêlée, semblent construire, au fil de

\footnotetext{
${ }^{15}$ L'agacement du joueur est lié au fait que son rôle est de contester le ballon en l'air. De son point de vue, il aurait dû sauter, tenter de récupérer le ballon ou, a minima, gêner son adversaire direct qui a capté le ballon.
} 
l'opposition, une intelligibilité de ce que l'arbitre montre. Ils ne partagent pas une compréhension de la situation, mais construisent, à partir de ce que montre l'arbitre, une compréhension de la manière dont ce dernier appréhende certaines actions -factuellement ou dans leur dynamique- et circonscrit ce qui est possible dans le jeu. Ce qui est progressivement partagé n'est alors pas de l'ordre de la signification de tel événement ou tel élément de contexte, mais du sens - de l'orientation- du déroulement du jeu. Même si cette hypothèse interprétative doit être mise à l'épreuve, nos résultats laissent entrevoir que, dans une situation d'interaction asymétrique ${ }^{16}$, la production d'une performance repose sur la construction d'un sens commun -au sens d'une orientation, d'une direction partagée- d'une part fondée sur une mise en visibilité de ce qui est acceptable et inacceptable, d'autre part dépendante du rapport cognitif de l'ensemble des participants à ce qui est montré comme tel.

\section{Evaluation de la prestation arbitrale et co-construction de la performance}

Si les résultats précédents établissent déjà que la performance arbitrale ne peut être réduire à la prestation d'un individu mais relève d'une co-production sur le terrain, il faut aussi considérer que l'activité de l'arbitre et ses modalités de coordination avec les joueurs ne sont pas indépendantes d'un contexte organisationnel et de normes culturelles. Comme le proposent Wisner (1997) et Engelström (1987), il faut penser le sujet et sa transaction avec le monde en intégrant la communauté, les outils, les règles et l'organisation collective. Il s'agit donc de considérer que la performance arbitrale n'est performance sans le jugement externe,

\footnotetext{
${ }^{16}$ Les situations d'interaction asymétrique recouvrent de notre point de vue des situations de co-action où une personne, responsable du déroulement de l'activité, peut et doit montrer aux autres ce qui est possible. Un enseignant, désigné par l'Education Nationale, montre à ses élèves ce qui est possible -et ce qui est valorisédans le contexte de classe, comme le fait aussi un entraîneur. Un cadre, nommé par l'entreprise, montre et impose à ses subordonnés une manière d'agir ensemble, de conduire un projet... un champ d'actions possibles dans ce contexte à un moment donné. Au-delà de l'arbitrage sportif, ces résultats invitent ainsi à ré-envisager les modalités de co-construction de la performance dans d'autres contextes d'activité.
} 
porté par le système sportif, sur la prestation arbitrale sur le terrain; ceci implique de développer une perspective plus systémique intégrant notamment la manière dont la performance est évaluée. Nous nous sommes donc attachés à investiguer l'activité des superviseurs chargés, par la fédération, d'apprécier la prestation arbitrale au cours d'une rencontre. Mais dans la mesure où l'arbitrage, en particulier l'arbitrage de haut niveau, est une prestation publique, nous nous sommes également intéressés à une évaluation profane publique à travers des articles de presse. Ces deux investigations visent à identifier les critères d'évaluation et les attentes qui pèsent sur la performance arbitrale de haut niveau. Même si nous ne pouvons mettre en évidence l'influence effective d'un critère ou d'une modalité d'évaluation sur la performance arbitrale co-produite sur le terrain, notre démarche suit celle des travaux en ergonomie de l'activité et/ou en psychologie du travail qui soulignent l'intérêt d'étudier les attentes du système. Ainsi, Caroly et Weill-Fassina (2004) ont montré, dans le domaine des services, l'impact sur la performance et la santé des opérateurs de la diversité des attentes auxquelles ils doivent faire face. Le vendeur en ligne, guichetier ou conseiller est souvent tiraillé entre la satisfaction des attentes de l'institution, celle des attentes des coacteurs de la prestation et ce qu'il considère comme devant être fait (Motté et Haradji, 2007). L'impossibilité de satisfaire la diversité et l'hétérogénéité des attentes relatives une prestation conduisent à des conflits d'évaluation : satisfaire l'ensemble des destinataires de son activité (y compris soi) est alors impossible et des choix difficiles doivent alors être faits sans qu'aucun bon choix ne soit possible. Ces situations d'injonctions contradictoires sont connues pour être à l'origine de problèmes de performance et de santé au travail (Coutarel, 2011).

\subsection{L’évaluation par le superviseur de la performance arbitrale : approcher les attentes de l'institution}


Pour étudier comment les institutions fédérales établissent la performance arbitrale et pour identifier ses standards de qualité par rapport à l'arbitrage, nous avons choisi d'investir le travail des superviseurs dont le rôle est de donner, pour un match particulier, une valeur à l'arbitrage réalisé. Nommé quelques jours avant la rencontre, le superviseur vient sur le lieu du match pour apprécier la performance de l'arbitre. Une fois le match terminé, il indique à l'arbitre les éléments positifs et négatifs de sa prestation et rédige in fine un rapport adressé à la fédération. L'ensemble des rapports effectués tout au long d'une saison sportive participe au classement des arbitres les uns par rapport aux autres; le résultat de l'évaluation du superviseur représente un enjeu pour les arbitres qui souhaitent accéder ou se maintenir au plus haut niveau. Afin d'approcher les standards de qualité à l'œuvre dans l'évaluation de la prestation de l'arbitre, nous nous sommes intéressés à la manière dont un superviseur évalue effectivement la prestation de l'arbitre.

Quatre matches ont été étudiés : trois en Top 14, un en Pro D2, le niveau inférieur ; quatre superviseurs différents ont contribué à ce travail au cours de la saison 2010/11. Pour chaque investigation, le match faisant l'objet de l'étude a été défini en collaboration avec la Fédération Française de Rugby ${ }^{17}$.

\footnotetext{
${ }^{17}$ Lors de chaque rencontre, une observation a été menée en accompagnant le superviseur tout au long de son activité, différentes traces d'activité du superviseur ont été collectées (prise de notes, grille d'observation, vidéo travaillée après le match) et trois entretiens ont été conduits avec chaque superviseur. Le premier a été réalisé avant le match. Son objectif était de renseigner la manière dont le superviseur s'y prend en match pour réaliser son évaluation de la prestation de l'arbitre. C'est à cette occasion, que chaque superviseur nous a présenté les outils utilisés pendant le match -grille d'observation officielle ou adaptée, dictaphone...- et les différents temps de son activité. Le second entretien, mené à la fin du match, incitait le superviseur à nous livrer ses impressions, son appréciation de la prestation de l'arbitre et du contexte de la rencontre. Il permettait également au chercheur de comprendre le travail que le superviseur effectuait ensuite : les modalités selon lesquelles il pensait revisionner le match à partir de supports vidéo fournis par les diffuseurs, la temporalité de son travail d'écriture du rapport... A partir de ces différents éléments, nous avons construit un journal de bord détaillé rendant compte de l'activité du superviseur tout au long du match. L'ultime entretien téléphonique avec le superviseur avait lieu une fois le rapport remis à la fédération et transmis à l'équipe de recherche. Cet entretien avait notamment pour objectif de proposer au superviseur notre interprétation de son activité.
} 
Le travail mené montre la complexité de l'établissement de la performance arbitrale (Boyer et al., 2012). Si le rapport produit par le superviseur caractérise in fine la prestation de l'arbitre au cours d'un match, nous avons essayé de saisir les standards de qualité sous-jacents à ce processus et de formaliser les attentes de l'institution.

Pendant le match, le superviseur produit à la fois une description fine du déroulement de la rencontre et des jugements évaluatifs ponctuels, ciblés sur des moments précis. Un superviseur mentionne d'une part tous les événements du match dans une grille d'observation ou en utilisant un support audio -mêlées, touches, mauls, irrégularités dans le jeu courant...-, d'autre part ses jugements évaluatifs ponctuels sur une feuille volante. Les événements auxquels il attribue une valeur positive ou négative sont ainsi directement identifiés sur ce second support. Ainsi, si tous les événements du match sont relevés, tous ne font pas l'objet d'une évaluation, seuls certains prennent d'emblée une connotation positive ou négative par rapport à l'arbitrage. La construction de la performance arbitrale résulte ensuite des liens logiques que les superviseurs construisent entre leurs jugements évaluatifs locaux. L'évaluation de la performance arbitrale ne se réduit donc pas à la somme de réussites ou d'échecs relatifs à des épisodes particuliers. La performance est établie dans des jugements de pratique globaux relativement à la continuité ou aux fluctuations des jugements évaluatifs locaux - positifs ou négatifs- liés à des types de situations. Ces jugements de pratique globaux instituent alors des situations comme exemplaires de performance ou de contre-performance. Ainsi, tout en s'ancrant dans des jugements évaluatifs locaux, la performance arbitrale se construit dans une historicité. La mise en évidence de ce processus permet de souligner une dialectique sous-jacente à l'évaluation de la performance arbitrale : cette dernière est établie en fonction des valeurs attribuées à des épisodes particuliers, mais procède également d'une appréciation globale de l'arbitrage tout au long de la rencontre. Si cette dialectique ne produit pas nécessairement de contradictions, elle génère de l'incertitude relativement à l'impact, sur 
l'évaluation, de tel ou tel jugement par rapport à tel ou tel épisode du match. Par exemple, l'attribution d'un carton peut faire l'objet d'un jugement local positif à l'instant $\mathrm{t}$, puis devenir une situation exemplaire de performance ou inversement de contre-performance selon les jugements locaux ultérieurs. Si les attentes de l'institution sont plutôt relatives à la qualité de l'arbitrage tout au long du match, il reste que l'arbitre ne peut pas anticiper le poids de ce qui peut être considéré comme une erreur ponctuelle.

L'étude de l'activité du superviseur a également permis de mettre en évidence le caractère crucial des premiers jugements évaluatifs locaux du superviseur dans la mesure où ils semblent orienter les suivants. En effet, ils amorcent des focalisations particulières et orientent le travail d'enquête constitutif de la supervision. Par exemple, si lors du signalement des premières fautes le superviseur attribue une valeur négative à la gestuelle de l'arbitre, celle-ci va faire l'objet d'une focalisation particulière dans la poursuite du match et dans l'évaluation de la prestation de l'arbitre. Il ne s'agit pas d'une impression que le superviseur va chercher à conforter mais d'une focalisation qui se construit en fonction de ses premiers jugements évaluatifs locaux. Ainsi, l'évaluation ne se réduit pas à une appréciation selon des critères totalement prédéterminés, mais les éléments appréciés lors de la supervision sont contextualisés au sens où ils se construisent au fil des jugements évaluatifs locaux qui orientent la(les) focalisation(s) future(s) du superviseur. Ce processus constitue une véritable contextualisation de l'évaluation : ce qui fonde un arbitrage de qualité est indexé au contexte. Mais ce faisant, les éléments appréciés, donc les attentes de l'institution, peuvent apparaître variables et peu explicites; le risque étant une difficulté pour l'arbitre de saisir ce que l'institution valorise et attend principalement de lui. Ce qui est en jeu en termes de performance et de santé, ce n'est pas nécessairement la définition de la «bonne pratique » mais plutôt la mise à jour et la possibilité de discussion collective des conceptions du « travail 
bien fait» pour établir temporairement un compromis acceptable par tous, et donc rediscutable $(\text { Clot, 2010) })^{18}$.

Enfin, l'étude des modalités d'évaluation de la prestation de l'arbitre permet de mettre en évidence que la définition de la qualité convoque des éléments subjectifs et des éléments objectifs. En effet, les jugements évaluatifs du superviseur se fondent tant sur un processus de valuation, où s'origine l'attribution spontanée de valeur, que sur la mobilisation d'éléments factuels qu'il relève dans sa description fine du déroulement du match. Les superviseurs partagent entre eux certains intérêts -c'est-à-dire des centrations relativement à ce qui importe- qui fondent leur attribution spontanée de valeur: (1) les protocoles utilisés par l'arbitre : par exemple, la manière dont il donne ses commandements en mêlée ; (2) le rapport de force dans le jeu : les superviseurs attribuent spontanément une valeur au rapport de force dans le jeu, à l'appréhension qu'en a l'arbitre et à sa gestion de ce rapport de force ; (3) les principes éthiques : les superviseurs appréhendent spontanément « ce qui se fait» ou « ne se fait pas» sur un terrain de rugby tant en termes de pratiques -certaines étant d'emblée déloyales et/ou dangereuses-, qu'en termes de communication entre l'arbitre et les joueurs relativement à leurs statuts respectifs. D'autres intérêts qui sont moins communément partagés, participent également à l'attribution spontanée de valeur. Des éléments objectivés sont ensuite convoqués et viennent supporter ou mettre en doute les jugements subjectifs spontanés émanant des intérêts de l'évaluateur. Ce dernier résultat montre que l'évaluation mobilise des éléments objectivés que tout un chacun est en mesure d'appréhender de manière identique - nombre de pénalités, de cartons, de turn-over ${ }^{19} \ldots$ - et des jugements subjectifs qui dépendent du partage, entre les membres de la communauté, d'intérêts, c'est-à-dire du partage de ce qui importe, de ce qui est premier.

\footnotetext{
${ }^{18}$ C'est l'existence et la dynamique du processus de débats autour des critères d'évaluation de la performance qui peut conduire à une amélioration de celle-ci, davantage que le produit nécessairement éphémère de ces débats (Clot, 2010).

${ }^{19}$ Les turn-over sont les changements de possession lors d'une phase de jeu au sol : l'équipe qui contrôle le ballon avant et après la phase de jeu au sol n'est plus la même.
} 
Cette étude de l'activité du superviseur montre que l'évaluation de la performance arbitrale se construit dans des dialectiques entre : (1) des valeurs attribuées à des épisodes particuliers et une appréciation globale de la prestation arbitrale tout au long de la rencontre, (2) la mobilisation de critères posés a priori et de focalisations construites en situation, (3) des jugements subjectifs et la convocation d'éléments objectivés. De ce fait, les attentes qui pèsent sur la prestation sur le terrain semblent difficiles à formaliser et variables. En effet, nous ne mettons pas en évidence des critères « objectifs » de performance, mais la complexité du processus d'évaluation de la performance. La performance arbitrale telle qu'elle est établie par l'institution se construit en considérant des éléments de nature opposée. Nous n'avons pas étudié la manière dont la considération de ces contraires et les incertitudes qu'elle génère pèsent effectivement sur la production arbitrale, mais au cours des entretiens, les verbatim de certains arbitres montrent que les attentes du superviseur sont prégnantes en situation : «Là "Main sur le ballon" c'est par rapport à un joueur qui est monté limite. Et là, c'est pas pour moi... C'est pas pour le public (...), c'est pour le superviseur. C'est-à-dire que le joueur, il a les mains sur le ballon, au cas où, il [le superviseur] aurait un doute, puisque l'autre il est monté limite... Comme il avait la main sur le ballon, je... c'est bon ». Il serait donc nécessaire d'étudier si, et le cas échéant comment, ces modalités d'évaluation et les attentes institutionnelles participent de la co-production d'une performance arbitrale sur le terrain.

\subsection{L'évaluation par la presse de la performance arbitrale : approcher les représentations sociales de l'arbitrage et les attentes du public}

Même si la pertinence de l'évaluation de la performance arbitrale effectuée dans les médias est souvent contestée par les instances arbitrales -et plus largement par le corps 
arbitral-, cette évaluation profane mais publique contribue à la construction de la performance arbitrale. Même si nous ne les analysons pas ici, plusieurs cas, dans différents sports, ont permis d'appréhender, ces dix dernières années, le poids de l'évaluation de la performance arbitrale par les médias. L'arbitre posé comme responsable d'une prestation arbitrale jugée négativement par la presse peut, quelques temps, être désigné pour officier des rencontres dont l'enjeu parait moindre et/ou qui font l'objet d'une diffusion plus réduite. L'image de l'arbitre, voire sa carrière, peuvent être durablement affectées à la suite d'une évaluation médiatique négative. Le travail effectué ici sur un corpus de presse n'analyse pas les effets des productions médiatiques sur la carrière de tel ou tel arbitre même si ces derniers ne peuvent plus être ignorés aujourd'hui. Ce travail exploratoire tente plutôt d'identifier les normes culturelles et les représentations sociales sous-jacentes à l'évaluation de la performance arbitrale, ainsi que les attentes qu'elles véhiculent. Si chacun s'approprie l'arbitrage et en a une appréhension particulière, les médias reprennent, construisent et alimentent les représentations sociales de l'arbitrage. L'analyse d'un corpus de publications parues dans le journal Midi Olympique a semblé propice à l'analyse des normes culturelles pesant sur la performance arbitrale en rugby dans la mesure où cet hebdomadaire se pose en défenseur de la culture rugbystique ${ }^{20}$. Là encore, nous ne sommes pas en mesure de mettre en évidence ce que les attentes du public génèrent relativement à la co-production de la performance arbitrale, mais notre observation participante inscrite dans la durée nous a souvent confronté à un adage ayant cours dans le milieu de l'arbitrage : « mieux vaut avoir tort avec tout le monde que raison tout seul ». L'investigation des normes culturelles et des représentations sociales sous jacentes à l'évaluation publique de la performance arbitrale peut ainsi s'avérer pertinente.

\footnotetext{
${ }^{20}$ Le Midi Olympique est un hebdomadaire, un journal de proximité dont le lectorat est composé de pratiquants et d'anciens pratiquants. Sa rédaction est largement composée de journalistes appartenant à la famille du rugby : il construit « une "communauté d'interprétation" forte dans la mesure où, en particulier sur les questions vives, il réagit souvent sinon de façon communautaire, du moins en souhaitant porter le débat devant la communauté et en insistant sur le caractère communautaire de ses propres interprétations » (Bonnet et Boure, 2008, p4).
} 
Afin de comprendre les normes culturelles et les représentations sociales sous-jacentes à la construction de la performance arbitrale lors de matches particuliers, nous nous sommes centrés sur les 18 comptes rendus de match ${ }^{21}$. La totalité porte un jugement négatif sur la prestation de l'arbitre : «arbitrage médiocre », « plusieurs décisions litigieuses », l'arbitrage est « énigmatique », « incompréhensible »... Certaines de ces appréciations se fondent sur des événements ponctuels où ce que montre l'arbitre est contesté par les entraineurs et/ou les présidents de clubs interviewés par le Midi Olympique : «Si je trouve cette pénalité sévère? Non je pense qu'elle n'a pas lieu d'être. C'est un maillot blanc qui bloque le ballon. Nous pour ça nous avons été sanctionnés cinquante fois et voilà qu'à la dernière minute, il siffle contre le porteur du ballon $»^{22}$. Ou encore « un essai entaché d'au moins un en avant $»^{23}$. Ce sont principalement des faits de jeu dont l'importance apparaît cruciale pour expliquer le résultat du match : un essai marqué ou refusé, une pénalité accordée ou non, ou encore un carton infligé à tel ou tel joueur. Mais dans d'autres publications, le jugement négatif est plus global, il repose sur l'identification des secteurs de jeu qui sont mal arbitrés. Les phases de jeu au sol, la mêlée et le maul sont en particulier ciblés : «il va falloir qu'on me réexplique l'arbitrage des rucks $»^{24}$, « ce soir, il ne sanctionne pas le pilier gauche qui met la main au sol à chaque mêlée $»^{25}$. Dans ce cas, deux arguments sont mobilisés : soit comme le reprend un article des « états généraux de l'arbitrage », il apparait que « la non-application de la règle est

\footnotetext{
${ }^{21}$ Nous avons inventorié tous les énoncés sur les arbitres ou l'arbitrage parus dans le Midi Olympique durant la saison 2010/2011, c'est-à-dire entre le 13 aout 2010 et le 4 juin 2011. Soixante-treize publications ont été relevées : 18 comptes rendus de match, 8 publications concernant un arbitre, 14 brèves mettant en avant des actions de formation, 10 articles constitués en dossier sur "Les états généraux de l'arbitrage » lancés par la rédaction du Midi Olympique en Avril/Mai 2011 et 19 publications parues dans la rubrique «courriers des lecteurs ».

${ }^{22}$ Midi Olympique du 17 Janvier 2011, propos tenus par un entraineur.

${ }^{23}$ Midi Olympique du 13 Mai 2011, événement rapporté par le journaliste pour abonder dans le sens des craintes d'un entraineur « J'ai bien sûr peur de l'arbitrage ».

${ }^{24}$ Midi Olympique du 7 Mars 2011, propos tenus par un entraineur.

${ }^{25}$ Midi Olympique du 4 Février 2011, propos tenus par un entraineur.
} 
la gangrène du rugby moderne $»^{26}$, soit c'est le déficit de connaissances techniques qui est reproché aux arbitres.

Ainsi, les jugements négatifs produits à l'encontre de l'arbitrage se fondent soit sur la convocation d'un épisode ponctuel qui est relevé au regard de son importance vis-à-vis du score, soit sur une appréciation plus globale de l'arbitrage relativement à une phase de jeu en particulier. Dans ce dernier cas, les attentes semblent relativement divergentes : soit l'arbitre doit mieux connaitre et appliquer la règle, soit l'arbitre doit faire preuve d'une meilleure connaissance et compréhension technique.

Cette étude exploratoire met en évidence le caractère exclusivement négatif de l'évaluation publique de la performance arbitrale. Elle ne pointe que des erreurs, des lacunes, des défaillances; cette tendance serait-elle révélatrice de représentations sociales valorisant un arbitrage «zéro défaut »? Cette hypothèse interprétative est renforcée par le fait que ce sont souvent les erreurs lors d'épisodes particuliers, considérés comme cruciaux, qui fondent la construction de la performance arbitrale dans les médias. Nous retrouvons malgré tout au cœur de la construction de la performance la dialectique entre des valeurs attribuées à des épisodes particuliers et une appréciation globale de la prestation arbitrale tout au long de la rencontre. Enfin, l'étude de ce corpus permet de souligner que quelle que soit la complexité de la construction et de la production de la performance arbitrale, le corps arbitral doit en assumer seul la pleine et entière responsabilité dans l'espace public.

Les deux études menées sur l'évaluation de la performance arbitrale montrent sa complexité : elle repose sur la considération d'éléments de natures opposées et elle est

\footnotetext{
${ }^{26}$ Titre d'un article du Midi Olympique du 23 Mai 2011.
} 
traversée par des logiques contradictoires. Ces études permettent également de souligner que les attentes du système pesant sur la production, sur le terrain, de la performance arbitrale sont diverses, variables et difficiles à formaliser. Mais la manière dont ces attentes, leurs variabilités et leurs dissonances influencent effectivement la performance arbitrale coproduite sur le terrain -et plus particulièrement les modalités de co-production de la performance arbitrale- reste à analyser. Si plusieurs éléments empiriques et différentes perspectives théoriques laissent présager que la co-production de la performance arbitrale sur le terrain n'est pas indépendante de la construction par le système de la performance arbitrale évaluée, d'autres travaux doivent étudier finement, si, et le cas échant comment, les attentes du système sont prégnantes pour les acteurs, en particulier l'arbitre, sur le terrain.

\section{Conclusion}

La performance arbitrale n'est pas la performance d'un seul homme. Les différentes modalités de coordination joueurs-arbitre en match mettent à jour certains mécanismes de coproduction de la performance arbitrale sur le terrain. Cette co-production tient tant à l'activité de l'arbitre -la manière dont il appréhende l'activité des joueurs et dont il rend visible et lisible ce qui est acceptable/inacceptable pour lui- qu'à l'activité des joueurs -en particulier leurs rapports cognitifs à ce que l'arbitre montre. Si nos résultats éclairent plutôt ce qui se joue dans l'instant, ils permettent d'émettre des hypothèses interprétatives sur les dynamiques éventuelles de la co-production d'une performance arbitrale tout au long du match. Mais, nous avons également considéré la performance arbitrale dans un contexte global, dans le climat culturel qui contribue à la construire (Ollis, et al., 2006). Pour ce faire, nous avons étudié les modalités d'évaluation de la performance arbitrale par le superviseur d'une part, par la presse 
d'autre part. Nous montrons que la construction de la performance arbitrale évaluée repose sur la considération d'éléments de natures hétérogènes et que ceci participe à la diversité, la variabilité et la difficulté à formaliser les attentes du système. Il faut aussi noter que, pour la presse, la performance arbitrale est toujours isolée de l'activité des joueurs, c'est la prestation de l'arbitre qui est passée au crible et son impact sur la performance des joueurs. Les médias font ainsi peser la responsabilité de la production de la performance arbitrale sur l'arbitre bien que les travaux sur l'activité des acteurs en situation établissent son caractère co-construit.

La mise en évidence des caractères co-produit -sur le terrain- et co-construit -par l'évaluation du système- de la performance arbitrale conduit à ré-envisager le développement de la performance arbitrale selon deux versants: l'un centré sur les acteurs de la coproduction -formation et analyse de l'activité des arbitres et des joueurs-, l'autre orienté vers les supports organisationnels de la co-construction -élaboration et partage des standards de qualité, espaces de discussion des attentes et des manières de faire avec les différentes parties prenantes... Notre travail met ainsi en évidence le caractère collectif, complexe et systémique de la production et de la construction de la performance arbitrale et ouvre de nouvelles perspectives quant à son développement.

\section{BIBLIOGRAPHIE}

Arnaud, P., 1995. Une histoire du sport. La documentation française, Paris.

Bonnet, V., Boure R., 2008. Le rugby entre patrimoine, récit et communication. Le cas de Midi Olympique. Semen 26 [En ligne: http://semen.revues.org.sicd.clermontuniversite.fr/8472]. 
Bourbousson, J., Poizat, G., Saury, J., Sève, C., 2011. Cognition collective : partage de préoccupations entre les joueurs d'une équipe de basket-ball au cours d'un match. Le travail humain 74(1), 59-90.

Boyer, S., Rix-Lièvre, G., Récopé, M., 2014. Evaluer une pratique en situation. L’activité de supervision d'arbitres de rugby : entre attribution spontanée de valeur et délibération. SpiralE, 53 [En ligne : http://spirale-edu-revue.fr/spip.php?article1181].

Cahour, B., Licoppe, C., 2010. Confrontations aux traces de son activité. Compréhension, développement et régulation de l'agir dans un monde de plus en plus réflexif. Revue d'anthropologie des connaissances 4(2), 243-253.

Caroly, S., Weill-Fassina, A., 2004. Evolutions des régulations de situations critiques au cours de la vie professionnelle dans les relations de service. Le travail humain, 67(4), 305-332.

Catteeuw, P., Gilis, B., Wagemans, J., Helsen, W., 2010. Offside Decision Making of Assistant Referees in the English Premier League: Impact of Physical and Perceptualcognitive Factors on Match Performance. Journal of Sports Sciences 28(11), 471-481.

Clot, Y., 2010. Le travail à cœur. Pour en finir avec les risques psychosociaux. La découverte, Paris.

Coutarel, F., 2011. Quelle reconnaissance du travail réel, des tensions et déséquilibres dans la quête de la performance? In P. Chaudat, Muller, R. (Eds.), Les nouvelles organisations du travail : entre souffrance et performance. L'Harmattan, Paris, pp. 73-99.

Coutts, A. J., Reaburn, P. R., 2000. Time and Motion Analysis of the AFL Field Umpire. Journal of Science and Medicine in Sport 3 (2), 132-139.

Darbon, S., 2008. Diffusion des sports et impérialisme anglo-saxon. De l'histoire événementielle à l'anthropologie. Maison des Sciences de l'Homme, Paris.

Engeström, Y., 1987. Learning by expanding. Orienta-Konsultit, Helsinki.

Guillén, F., Feltz, D. L., 2011. A Conceptual Model of Referee Efficacy. Frontiers in Psychology 2 (25) [En ligne : http://dx.doi.org/10.3389/fpsyg.2011.00025]. 
Hauw, D., Durand, M., 2004. Pour une "dé-psychologisation" de la performance sportive de haut niveau. Movement \& Sport Sciences 53, 119-123.

Helsen, W., Bultynck, J.-B., 2004. Physical and Perceptual-cognitive Demands of Top-class Refereeing in Association Football. Journal of Sports Sciences 22, 179-189.

Kay, B., Gill, N., 2003. Physical Demands of Elite Rugby League Referees: Part One - Time and Motion Analysis. Journal of Science and Medicine in Sport 6(3), 339-342.

Kay, B., Gill, N., 2004. Physical Demands of Elite Rugby League Referees. Part To: Heart Responses and Implications for Training and Fitness Testing. Journal of Science and Medicine in Sport 7(2), 165-173.

Krustrup, P., Helsen, W., Randers, M. B., Christensen, J. F., MacDonald, C., Rebelo, A. N., Bangsbo, J., 2009. Activity Profile and Physical Demands of Football Referees and Assistant Referees in International Games. Journal of Sports Sciences 27(11), 1167-1176.

Licoppe, C., 2008. Dans le "carré de l'activité": perspectives internationales sur le travail et l'activité. Sociologie du travail 50(3), 287-302.

MacMahon, C., 1999. Making Sense of Chaos: Decision Making by High and Low Experience Rugby Referees. Rapport de recherche, University of Ottawa, Ottawa.

Mascarenhas, D. R. D., Collins, D., Mortimer, P., 2005. Elite Refereeing Performance: Developing a Model for Sport Science Support. The Sport Psychologist 19(4), 364-379.

Mascarenhas, R. D., Collins, D., Mortimer, P., Morris, R. L., 2005. A Naturalistic Approach to Training Accurate and Coherent Decision Making in Rugby Union Referees. The Sport Psychologist 19(2), 131-147.

Mascarenhas, R. D., O'Hare, D., Plessner, H., 2006. The Psychological and Performance Demands of Association Football Refereeing. International Journal of Sport Psychology 37(2-3), 99-120. 
Mellick, M., Flemming, S., Bull, P., Laugharne, E. J., 2005. Identifying Best Practice for Referee Decision Communication in Association and Rugby Union Football. Football Studies $8,42-57$.

Mellick, M., Flemming, S., Davies, G., 2007. An Interpretive Analysis of Interpersonal Communication: a Case Study from Elite Rugby Union Match Officiating. International Journal of Performance Analysis in Sport 7(2), 92-105.

Motté, F., Haradji, Y., 2007. Le vendeur en ligne dans la relation de service. Communication au 42ème congrès de la SELF, Ergonomie des produits et des services, Toulouse.

Ollis, S., Macpherson, A., Collins, D., 2006. Expertise and Talent Development in Rugby Refereeing: An Ethnographic Enquiry. Journal of Sport Sciences 24 (3), 309-322.

Oudejans, R. R. D., Bakker, F. C., Verheijen, R., Gerrits, J. C., Steinbruckner, M., Beek, P. J., 2005. How Position and Motion of Expert Assistant Referees in Soccer Relate to the Quality of their Offside Judgments during Actual Match Play. International Journal of Sport Psychology 36 (1), 3-21.

Plessner, H., Haar, T., 2006. Sports Performance Judgments from Social Cognitive Perspective. Psychology of Sport and Exercise 7 (6), 555-575.

Poizat, G., Bourbousson, J., Saury, J., Sève, C., 2012. Understanding Team Coordination in Doubles Table Tennis: Joint Analysis of First- and Third-person Data. Psychology of Sport and Exercise 13 (5), 630-639.

Reynes, E., Canovas, S., Ferrand, C., Pantaleon, N., 2008. Conséquences émotionnelles des erreurs d'arbitrage chez les footballeurs: étude exploratoire. Psicologia \& Sociedade 20(1), $5-15$.

Rix, G., 2005. Typologie des actes de jugement de l'arbitre de rugby expérimenté. Science et Motricité 56(3), 109-124. 
Rix-Lièvre, G., 2010. Différents modes de confrontation à des traces de sa propre activité. Entre convergences et spécificités. Revue d'anthropologie des connaissances 4(2010/2), 357-376.

Rix-Lièvre, G., Boyer, S., Coutarel, F., Lièvre, P., 2014. La performance arbitrale : de son étude à son développement. Activités 11(1), 86-104

Rix, G., Biache, M-J., 2004. Enregistrement en perspective subjective située et entretien en re situ subjectif : une méthodologie de constitution de l'expérience. Intellectica 38, 363-396.

Salembier, P., Zouinar, M., 2004. Intelligibilité mutuelle et contexte partagé, Inspirations conceptuelles et réductions technologiques. @ctivités 1 (2), 64-85.

Serres, M., 2010. Les arbitres ne font pas d'erreur. In R. Lefebvre, Jouve, F. (Eds.), Tous arbitres. Editions Chronique, Paris, pp. 8-15.

Sève, C., Poizat, G., Saury, J., Durand, M., 2006. Un programme de recherche articulant analyse de l'activité en situation et conception d'aides à la performance: un exemple en entraînement sportif de haut niveau. @ctivités 3 (2), 46-64.

Simmons, P., 2007. Fair Call: Player Perceptions of Justice in Football Referee Communication. Colloque « Communications, Civics, Industry », Melbourne University.

Theureau, J., 1992. Le cours d'action, analyse sémiologique : essai d'une anthropologie cognitive située. Peter Lang, Berne.

Valléry, G., Leduc, S., 2005. Contribution ergonomique à l'analyse des relations de service. Exemple de professionnalisation d'une fonction d'accueil en bureau de poste. Travail Humain 68(2), 153-189.

Vermersch, P., 1994. L'entretien d'explicitation. ESF, Paris.

Wisner, A., 1997. Aspects psychologiques de l'anthropotechnologie. Travail Humain 60(3), 229-254. 